K. F. Lai

Nagoya Math. J.

Vol. 85 (1982), 155-174

\title{
ON THE COHOMOLOGY OF CONGRUENCE SUBGROUPS OF SYMPLECTIC GROUPS
}

\author{
K. F. LAI
}

\section{$\S 1$. Introduction}

This paper is concerned with the cohomology at "infinity" (in the sense of Harder [4], [5]) of a congruence subgroup of the symplectic group $G=S p(2 \ell, R) . \quad G$ is the subgroup of $G L(2 \ell, R)$ consisting of matrices $g$ satisfying ${ }^{t} g J g=J$ where

$$
J=\left(\begin{array}{rr}
0 & I \\
-I & 0
\end{array}\right)
$$

and $I$ is the $\ell \times \ell$ identity matrix. We consider $G$ as the real points of the algebraic group $\underline{G}=S p(2 \ell)$ defined over $\boldsymbol{Q}$. Let $p$ be a prime not equal to 2 and $\Gamma$ be the kernel of the natural map

$$
S p(2 \ell, Z) \longrightarrow S p\left(2 \ell, Z / p^{r} Z\right) \text {. }
$$

We assume that $r$ is chosen large enough so that $\Gamma$ is torsion free.

We are interested in the Eilenberg-Maclane cohomology groups $H^{*}(\Gamma, C)$ of $\Gamma$ (cf: Borel [2]). It is well-known that $H^{*}(\Gamma, C) \approx H^{*}(X \mid \Gamma, C)$ where $X$ is the symmetric space of maximal compact subgroups of $G$ and $G$ acts in a natural way on $X$. In [3] Borel and Serre constructed a compactification $\bar{X} / \Gamma$ of $X / \Gamma$ having the property that $H^{*}(\bar{X} / \Gamma, C) \approx H^{*}(X / \Gamma, C)$. $\bar{X} / \Gamma$ is a manifold with corners and is a union of subsets $e^{\prime}(P)$ (see [3] p. 476) where $P$ runs over the $\Gamma$ conjugacy classes of parabolic $Q$-subgroups of $\underline{G}$. Let

$$
r: H^{*}(\bar{X} / \Gamma, C) \longrightarrow H^{*}(\partial(\bar{X} / \Gamma), C)
$$

be the homomorphism induced by the map $\partial(\bar{X} / \Gamma) \rightarrow \bar{X} / \Gamma$. The general programme is to investigate the existence of a subspace $H_{\mathrm{inf}}^{*}(\Gamma, C)$ of $H^{*}(\Gamma, C) \approx H^{*}(\bar{X} / \Gamma, C)$ which restricts isomorphically onto $\operatorname{Im} r$. The 
elements of $H^{*}{ }_{\text {inf }}(T, C)$ can be considered as describing the cohomology at "infinity".

Fix a standard Borel subgroup $B$ of $G$ (see $\S 1$ ). Let $T$ be the maximal torus in $B$ and $m$ be the dimension of the unipotent radical of $B$. Let $D$ be a set of representatives for all the $\Gamma$-conjugacy classes of minimal parabolic subgroups of $G$ which are defined over $\boldsymbol{Q}$. Choose $D$ so that $B \in$ $D$. Following $G$. Harder we use Langlands' theory of Eisenstein series to construct elements of $H_{\text {inf }}^{*}(T, C)$ from cohomology classes in $\oplus_{P \in D} H^{*}\left(e^{\prime}(P)\right.$, $C)$. For precise statement see $\S 10$.

In the case of $\boldsymbol{Q}$-rank one algebraic groups, Harder has shown that cohomology classes constructed this way exhaust the entire space $H_{\text {inf }}^{*}(T, C)$ (cf: [4], [5]). Schwermer has obtained similar results for $S L(3)$ and $S L(n)$ (cf: [11], [12]).

Notation. For an algebraic subgroup $\underline{G}$ of $G L(n)$ defined over a field $k$ and $r$ a subring of $k, \underline{G}(r)$ denotes the subgroup of $\underline{G}(k)$ (the group of $k$-rational points of $\underline{G}$ ) consisting of $n \times n$ matrices with entries in $r$ and determinants units in $r$.

For a prime $p$ of the rational numbers $\boldsymbol{Q}$ we write $\boldsymbol{Q}_{p}$ (resp. $Z_{p}$ ) for the $p$-adic numbers (resp. integers). $\boldsymbol{A}$ (resp. $\boldsymbol{A}_{0}$ ) denotes the adeles (resp. the finite adeles) of $\boldsymbol{Q}$.

$\S 2$.

Let $\mathfrak{g}$ be the Lie algebra of $G ; \mathfrak{g}$ consists of $2 \ell \times 2 \ell$ real matrices $X$ satisfying the condition: ${ }^{t} X J+J X=0$. Let $a$ be the Cartan subalgebra of $g$ consisting of real matrices

$$
H=\left(\begin{array}{lllllll}
h_{1} & & & & & \\
h_{2} & & & & \\
& \ddots & & & \\
& & h_{\ell} & & \\
& & -h_{1} & & \\
& & & -h_{2} & & \\
& & & & \ddots & \\
& & & & & -h_{\ell}
\end{array}\right) .
$$

For $1 \leq j \leq \ell$, define elements $\varepsilon_{j}$ in the dual space $\mathfrak{a}^{*}$ of $\mathfrak{a}$ by $\varepsilon_{j}(H)=h_{j}$. Then the root system of the pair $(\mathfrak{g}, \mathfrak{a})$ is given by

$$
\Psi=\left\{ \pm \varepsilon_{i} \pm \varepsilon_{j}: i \neq j, 1 \leq i, j \leq \ell\right\} \cup\left\{ \pm 2 \varepsilon_{j}: 1 \leq j \leq \ell\right\}
$$


and the set of simple roots is

$$
\Delta=\left\{\varepsilon_{1}-\varepsilon_{2}, \varepsilon_{2}-\varepsilon_{3} \cdots, \varepsilon_{\ell-1}-\varepsilon_{\ell}, 2 \varepsilon_{\ell}\right\}
$$

we also write $\alpha_{j}=\varepsilon_{j}-\varepsilon_{j+1}$ for $1 \leq j \leq \ell-1$ and $\alpha_{\ell}=2 \varepsilon_{\ell}$.

For $\mu \in \mathfrak{a}^{*}, H \in \mathfrak{a}$ we denote with $\langle H, \mu\rangle$ the value $\mu(H)$. The restriction of the Killing form $(\cdot, \cdot)$ of $\mathfrak{g}$ to $\mathfrak{a}$ is nonsingular and hence one can define a map $\mu \rightarrow H(\mu)$ of $\mathfrak{a}^{*}$ onto $a$ by the relation

$$
(H, H(\mu))=\langle H, \mu\rangle,
$$

for all $H \in \mathfrak{a}$. The mapping defines a nonsingular bilinear form on $a^{*}$ given by

$$
(\mu, \lambda)=\langle H(\mu), \lambda\rangle \text {. }
$$

Denote by $g_{\alpha}$ the root subspace of $g$ corresponding to the root $\alpha$. Let $\Psi^{+}$ (resp. $\Psi^{-}$) be the set of positive (resp. negative) roots with respect to the order determined by $\Delta$. Let

$$
\mathfrak{n}=\sum_{\alpha \in \mathbb{\Psi}} \mathfrak{g}_{\alpha}
$$

and $\mathfrak{b}=\mathfrak{a}+\mathfrak{n}$, the Borel subalgebra of $\mathfrak{g}$ containing $\mathfrak{a}$. Let $B$ be the Borel subgroup of $G$ with Lie algebra $\mathfrak{b}, N$ be the unipotent radical of $B$ and $T$ the maximal torus of $G$ in $B$. Then $B=T . N$. Let $K$ be the intersection of $G$ and the special orthogonal group $S O(2 \ell, R) . \quad K$ is a maximal compact subgroup of $G$ consisting of matrices

$$
\left(\begin{array}{rr}
X & Y \\
-Y & X
\end{array}\right)
$$

satisfying the relations

$$
{ }^{t} X X+{ }^{t} Y Y=1, \quad{ }^{t} X Y={ }^{t} Y X .
$$

Moreover $G=K . B=K A N$ (Iawasawa decomposition), where $A$ is the identity component of $T=\underline{T}(R)$.

$X$ can be identified with $K \backslash G$ and we have a principal fibration

$$
\pi: G / \Gamma \longrightarrow X \mid \Gamma
$$

with structure group $K$. Let $x_{0}$ be the point in $X$ fixed by $K$. Then we can identify the tangent space at $x_{0}$ with $\mathfrak{b}$ and the tangent bundle of $X / \Gamma$ is the bundle induced (via $\pi$ ) by adjoint representation $\mathrm{Ad}$ of $K$ on $\mathfrak{b}$ ([5] p. 131). 
Let $\Omega^{m}(X \mid \Gamma)$ be the vector space of smooth $C$-valued differential $m$ forms on $X / \Gamma$. Then the cohomology groups $H^{*}(X \mid \Gamma, C)$ are canonically isomorphic to the cohomology groups of the de Rham complex $\left(\Omega^{*}(X / \Gamma), d\right)$ (cf: [5], [9]). Moreover there is a natural identification between the space $\Omega^{m}(X \mid \Gamma)$ and the space of smooth functions

$$
\phi: G / \Gamma \longrightarrow \operatorname{Hom}\left(\Lambda^{m} \mathfrak{b}, C\right)
$$

which satisfy

$$
\phi(k g)=\Lambda^{m} \operatorname{Ad}^{*}(k) \phi(g), \quad g \in G, \quad k \in K
$$

where $\mathrm{Ad}^{*}$ is the dual representation to the adjoint representation $\mathrm{Ad}$ of $K$ on $B$ (cf: [5] $\S 1$ ).

Matsushima-Murakami ([9]) defined an Laplacian operator $\Delta$ on the complex $\Omega^{*}(X / \Gamma)$. A form $\omega \in \Omega^{*}(X / \Gamma)$ is then said to be harmonic if $\Delta \omega$ $=0$. On the other hand, the universal enveloping algebra $U\left(g_{c}\right)$ of $g_{c}=$ $\mathrm{g} \otimes C$ operates as an algebra of differential operators on the smooth functions on $G / \Gamma$ with values in $\operatorname{Hom}\left(\Lambda^{m} \mathfrak{b}, C\right)$ ([6] chap I \& 2). The Casimir operator $C$ defined with respect to the Killing form lies in the centre $\mathscr{Z}$ of $U(\mathrm{~g})$ and it sends smooth functions satisfying (2.3) into smooth functions satisfying the same conditions. Moreover according to the lemma of Kuga ([9] §6) we have for all smooth $m$-forms $\phi: G / \Gamma \rightarrow \operatorname{Hom}\left(\Lambda^{m} \mathfrak{b}, C\right)$ the formula

$$
\Delta \phi=-C \phi
$$

$\S 3$.

Let $\underline{G}(A)$ be the adele group of $\underline{G}([14])$.

Let

$$
\begin{aligned}
& \Gamma_{p}=\left\{\left(\gamma_{q}\right) \in \underline{G}(A) \mid \gamma_{p} \in G\left(Z_{p}\right), \gamma_{p} \equiv 1 \bmod p^{r}, \text { and } \gamma_{q}=1 \text { if } q \neq p\right\} \\
& K_{0}=\prod_{p} \underline{G}\left(Z_{p}\right) \\
& K_{r}=\Gamma_{p} \prod_{q \neq p} \underline{G}\left(Z_{q}\right) \\
& \underline{G}=K_{r} \backslash K_{0} .
\end{aligned}
$$

Write $\underset{\sim}{B}($ resp. $\underset{\sim}{T}, \underset{\sim}{N})$ for the image of $\underline{B}\left(A_{0}\right) \cap K_{0}\left(\right.$ resp. $\underline{T}\left(A_{0}\right) \cap K_{0},\left( \pm 1 . \underline{N}\left(A_{0}\right)\right)$ $\left.\cap K_{0}\right)$ in $G$. Then it is a well-known consequence of the strong approximation theorem that the $\Gamma$-conjugacy classes of Borel $\boldsymbol{Q}$-subgroups ( $\Gamma$ as defined in $\S 1$ ) corresponds bijectively with the Cartesian product $X \times Y$ where $X=\underset{\sim}{B} \backslash \underline{G}$ and 


$$
Y=K_{0} \cap \underline{B}\left(A_{0}\right) \backslash \underline{B}\left(A_{0}\right) / \underline{B}(\boldsymbol{Q}) .
$$

One can then deduce the following

LEMMA 3.1. Let $\chi$ be a unitary character of $\underset{\sim}{T}$ satisfying

$$
\chi(t)=1
$$

for all $t$ in the image of $\underline{T}(\boldsymbol{Q}) \cap K_{r}$ in $\underline{T}$.

Let $V_{x}$ be the complex vector space of $C$-valued functions $f$ on $G$ which are right invariant under $\underset{\sim}{N}$ and satisfy

$$
f(k t)=\chi(t) f(k)
$$

for $k \in \underset{\sim}{G}$ and $t \in \underset{\sim}{T}$. Then there exists an isomorphism

$$
\oplus_{P \in D} H^{*}\left(e^{\prime}(P), C\right) \widetilde{\rightarrow} H^{*}\left(e^{\prime}(B), C\right) \otimes\left(\oplus_{\chi} V_{z}\right) .
$$

(The direct sum on the right is taken over all the unitary characters $\chi$ of T satisfying (3.1).)

The explicit form of this isomorphism is not needed here and so we will not reproduce here the proof of this lemma (which follows the same lines as Satz 5.7 and p. 40 of Schwermer [12]). As a consequence of Lemma 3.1, an element of $\oplus_{P \in D} H^{*}\left(e^{\prime}(P), C\right)$ can be identified with $\omega \otimes \sum f_{\text {, for }}$ some $\omega \in H^{*}\left(e^{\prime}(B), C\right)$ and $f_{x} \in V_{x}$. Next we seek to represent $\omega$ by a function on $G / B \cap \Gamma$.

$\S 4$.

According to Proposition 9.4 of Borel-Serre [3] we have

$$
e^{\prime}(B)=N / N \cap \Gamma \text {. }
$$

Thus

$$
H^{*}\left(e^{\prime}(B), C\right)=H^{*}(N / N \cap \Gamma, C) .
$$

The right hand side of (4.1) can be computed by using the de Rham complex $\Omega^{*}(N / N \cap \Gamma, C)$ and we can identify $\Omega^{m}(N / N \cap \Gamma, C)$ with the space of smooth functions $N \rightarrow \operatorname{Hom}\left(\Lambda^{m} \mathfrak{n}, C\right)$ which are right invariant under $N \cap \Gamma$. (Note that the tangent bundle of $N / N \cap \Gamma$ is trivial.) Thus a cohomology class of $H^{m}\left(e^{\prime}(B), C\right)$ can be represented by a smooth function $\phi$ on $N$ with values in $\operatorname{Hom}\left(\Lambda^{m} \mathfrak{n}, C\right)$. By composing with the embedding

$$
\operatorname{Hom}\left(\Lambda^{m} \mathfrak{n}, C\right) \longrightarrow \operatorname{Hom}\left(\Lambda^{m} \mathfrak{b}, C\right),
$$


we can think of $\phi$ as taking values in $\operatorname{Hom}\left(\Lambda^{m} \mathfrak{b}, C\right)$. For $\lambda \in \mathfrak{a}_{\boldsymbol{c}}^{*}$, we extend $\phi$ to a function

$$
\phi_{\lambda}: G / B \cap \Gamma \longrightarrow \operatorname{Hom}\left(\Lambda^{m} \mathfrak{b}, C\right)
$$

by

$$
\phi_{\lambda}(g)=\Lambda^{m} \operatorname{Ad}^{*}(k)(\phi(1)) \xi_{-\lambda-\rho}(a)
$$

if $g=k a n \in G=K A N, \rho=1 / 2 \sum_{\alpha \in \Psi+} \alpha$ and $\xi_{-\lambda-\rho}$ is the character on $\underline{T}$ associated to $-\lambda-\rho$.

$\S 5$.

Next we want to extend $\phi_{\lambda}$ to a function defined on $\underline{G}(A)$.

First we note that $\underline{G}(\boldsymbol{Q})$ is embedded as a discrete subgroup of $\underline{G}(\boldsymbol{A})$ and

$$
\Gamma=\underline{G}(\boldsymbol{Q}) \cap K_{r} .
$$

It is well-known that

$$
\underline{G}(A)=K \cdot K_{0} \cdot \underline{B}(A)
$$

and

$$
G / \Gamma=K_{r} \backslash \underline{G}(A) / \underline{G}(\boldsymbol{Q}) .
$$

(See for example [1]). In particular this means that any function on $K_{r} \backslash \underline{G}(A) / \underline{G}(\boldsymbol{Q})$ is determined by its restriction to $G / \Gamma$.

A function $f$ in $V_{x}$ (see $\S 3$ ) can be thought of as a function on $G$ right invariant under $\underset{\sim}{N}$. $K_{0}$ acts on the vector space $C(G)$ of complex valued functions on $\underset{\sim}{G}$ by left translation, $\underset{\sim}{L}$ :

$$
\underset{\sim}{L}(k) f(g)=f\left(k^{-1} g\right), \quad k \in K_{0}, \quad g \in \underset{\sim}{G} .
$$

Let $\mathscr{P}_{*}$ be the projection from $C(G)$ to $V_{x}$. Then $L(k) f=\mathscr{P}_{x} \underline{\sim}(k) f$ defines an action of $K_{0}$ on $V_{x}$. We can give $C(G)$ an inner product such that $L(k)$ is an unitary operator for all $k$.

The unitary character $\chi$ on $\underset{\sim}{T}$ can be trivially extended to an unitary character on

$$
\underline{T}(R) \cdot \underline{T}\left(A_{0}\right) \cap K_{r} \backslash \underline{T}(A) / \underline{T}(Q) .
$$

We use the same symbol $\chi$ for the extended character. Note that if $t=$ $\left(t_{p}\right) \in \underline{T}(A), t_{q} \in \underline{T}\left(Z_{q}\right)$ for $q \neq p$ and $t_{p}=1$, then $\chi(t)=1$. 
By $\S 3$ every element of $\oplus_{P \in D} H^{m}\left(e^{\prime}(P), C\right)$ corresponds to a $[\phi] \otimes \sum_{x} f_{x}$ for some cohomology class [ $\phi$ ] of $H^{m}\left(e^{\prime}(B), C\right)$ represented by $\phi$ and some $f_{x} \in V_{z}$. For $\lambda \in \mathfrak{a}_{c}^{*}$, we associate to $[\phi] \otimes f_{z}$ a function

$$
\Phi_{z}: \underline{G}(A) / \underline{B}(\boldsymbol{Q}) \longrightarrow \operatorname{Hom}\left(\Lambda^{m} \mathfrak{b}, C\right) \otimes V_{\gamma}
$$

given by

$$
\Phi_{z}(g)=\left(\Lambda^{m} \operatorname{Ad}^{*}(k) \phi(1)\right) \otimes\left(L\left(k_{0}\right) f_{z}\right) \gamma(t) \xi_{-\lambda-\rho}(t)
$$

if $g=k k_{0} t n \in \underline{G}(A)=K K_{0} \underline{T}(A) \underline{N}(A)$. Here, $\xi_{\lambda}$ is the character of $\underline{T}$ associated to $\lambda$ and $\xi_{\lambda}$ defines a character on $\underline{T}(A)$. We shall write $\Phi$ for $\Phi_{k}$ unless specified otherwise. The following lemma is a straightforward consequence of the definition.

Lemma 5.1. For $g=\left(g_{q}\right) \in \underline{G}(A)$ and $t \in \underline{T}(A)$ we have

(i) $\Phi(g t)=\Phi(g) \chi(t) \xi_{-\lambda-\rho}(t)$.

(ii) If $g_{q}=1$ for all finite prime $q$, then

$$
\Phi(g)=\phi_{\lambda}\left(g_{\infty}\right) \otimes f_{x} .
$$

(iii) If $g_{q^{\prime}}=1$ for all primes (including $\infty$ ) $q^{\prime}$ except one $q \neq p$ and $g_{q}=k_{q} t_{q} n_{q} \in \underline{G}\left(\boldsymbol{Q}_{q}\right)=\underline{G}\left(Z_{q}\right) \underline{T}\left(A_{q}\right) \underline{N}\left(A_{q}\right)$ then

$$
\Phi(g)=\chi\left(t_{q}\right) \xi_{-\lambda-\rho}\left(t_{q}\right)\left(\phi(1) \otimes f_{x}\right) .
$$

(iv) If $g_{q}=1$ for all primes (including $\infty$ ) except the prime $p$ and $g_{p}$, $=k_{p} t_{p} n_{p}$, then

$$
\Phi(g)=\left(\phi(1) \otimes L\left(k_{0}\right) f_{x}\right) \chi\left(t_{p}\right) \xi_{-\lambda-\rho}\left(t_{n}\right)
$$

where $k$ is the adele $\left(1, \cdots, 1, k_{p}, 1, \cdots, 1\right)$. Moreover, we have

$$
\|\Phi(g)\| \leq\left|\xi_{-\lambda-\rho}\left(t_{p}\right)\right| ! f_{x}\|\| \phi(1) \| .
$$

$\S 6$.

We recall some results on differential operators.

Let $W$ be the Weyl group of $(\mathfrak{g}, \mathfrak{a})$. For $w \in W$, let $\# w$ denote the number of elements in the set $w \Psi^{-} \cap \Psi^{+}$. For any nonnegative integer $m$ put

$$
W(m)=\{w \in W: \# w=m\} .
$$

The adjoint representation induces a representation of $a$ on $\operatorname{Hom}\left(\Lambda^{*} \mathfrak{n}, C\right)$ which commutes with the coboundary operator (cf: [7] $\S 5.7$ ) and so gives 
rise to a representation of $\mathfrak{a}$ on $H^{*}(\mathfrak{n}, C)$. For any weight $\mu$ of this representation, let $H^{*}(\mathfrak{n})^{\mu}$ be the space of all classes in $H^{*}(\mathfrak{n}, C)$ whose weight is $\mu$. Kostant [7] proved that

$$
H^{*}(\mathfrak{n}, C)=\oplus H^{*}(\mathfrak{n})^{\mu}
$$

where $\mu$ satisfies

$$
(\rho, \rho)=(\rho+\mu, \rho+\mu)
$$

and each $H^{*}(\mathfrak{n})^{\mu}$ is irreducible. Moreover

$$
H^{m}(\mathfrak{n}, C)=\oplus_{w \in W(m)} H(\mathfrak{n})^{w \rho-\rho} .
$$

The vector space $\operatorname{Hom}\left(\Lambda^{m} \mathfrak{n}, C\right)$ can be considered as the space of smooth functions $N \rightarrow \operatorname{Hom}\left(\Lambda^{m} \mathfrak{n}, C\right)$ which are invariant under $N$. Therefore we have an embedding

$$
\operatorname{Hom}\left(\Lambda^{*} \mathfrak{n}, C\right) \longrightarrow \Omega^{*}(N / N \cap \Gamma, C)
$$

(cf, §4). It is an easy consequence of the theorems of van Est [13] that the above embedding induces isomorphism on cohomology:

$$
H^{*}(\mathfrak{n}, C) \approx H^{*}(N / N \cap \Gamma, C)
$$

(cf: [5] Theorem 2.2). Corresponding to (6.1) we have the decomposition

$$
H^{*}\left(e^{\prime}(B), C\right)=\oplus H\left(e^{\prime}(B)\right)^{\mu} .
$$

The elements of $H\left(e^{\prime}(B)\right)^{\mu}$ are called cohomology classes of weight $\mu$. In particular if $w_{0}$ is the element of the Weyl group satisfying $w_{0} \Psi^{-}=\Psi^{+}$ then $\sharp\left(w_{0}\right)=\operatorname{dim} \mathfrak{n}$ and (6.3) yields

$$
H^{m}\left(e^{\prime}(B), C\right)=H\left(e^{\prime}(B), C\right)^{-2 \rho}, \quad m=\operatorname{dim} \mathfrak{n} .
$$

LEMMA 6.1.

(i) Every cohomology class in $H^{m}\left(e^{\prime}(B), C\right)$ can be represented by a harmonic differential form $\phi$.

(ii) If $\phi$ is chosen as in (i) and $\Phi_{x}$ is the function defined by (5.3) then the Eisenstein series

$$
E\left(g, \Phi_{\chi}\right)=\sum_{\underline{G}(\boldsymbol{Q}) / \underline{B}(\boldsymbol{Q})} \Phi_{\chi}(g \gamma)
$$

is a "smooth" function on $G(A)$ and is holomorphic (as a function of $\lambda$ ) in the domain defined by the condition

$$
\operatorname{Re}(\lambda, \alpha)>(\rho, \alpha) \quad \text { for all } \alpha \in \Psi^{+} .
$$


Moreover, $E\left(g, \Phi_{\chi}\right)$ has meromorphic continuation into the entire $\mathfrak{a}_{c}^{*}$.

(iii) If $\phi$ is chosen as in (i) and $E\left(g, \Phi_{\chi}\right)$ is holomorphic at $\lambda$ then $E\left(g, \Phi_{\chi}\right)$ defines a differential form on $X / \Gamma$. Moreover if $\phi$ is of weight $\mu$, then we have

$$
\Delta E\left(g, \Phi_{\chi}\right)=((\mu+\rho, \mu+\rho)-(\lambda, \lambda)) E\left(g, \Phi_{\chi}\right) .
$$

Proof. (i) follows trivially from the fact that $e^{\prime}(B)=N / N \cap \Gamma$ is compact. If $\phi$ is harmonic, then by Kuga's lemma (§2) $\phi$ is an eigenfunction of the Casimir operator. Moreover $\phi$ is trivially a cusp form, so we can apply the theorem of Borel-Garland ([2] Theorem 6.2) to conclude that $\phi$ is an automorphic form in the sense of Harish-Chandra, Langlands ([6], [8]).

(ii) now follows from standard results on Eisenstein series (cf: [6] Chap. $11 \S 2$, [8] Chap. 4 and Appendix II). It is clear from the definition of $f_{z}$ and (5.3) that $E\left(g, \Phi_{z}\right)$ is a function on $K_{r} \backslash \underline{G}(\boldsymbol{A}) / \underline{G}(\boldsymbol{Q})$. Thus it defines, via (5.2) a function on $G / \Gamma$ with values in $\operatorname{Hom}\left(\Lambda^{m} \mathfrak{b}, C\right) \otimes V_{x}$ and by $\S 2$ is a differential form on $X / \Gamma$. The formula (6.7) for the Laplacian operator is a trivial consequence of Kuga's lemma.

Suppose that under the map (3.3) the element $\omega$ of $\oplus_{P \in D} H^{*}\left(e^{\prime}(P), C\right)$ corresponds to $[\phi] \otimes \sum_{x} f_{x}$ where $\phi$ is chosen to be harmonic. Let $E(g, \omega)$ be $\sum_{x} E\left(g, \Phi_{\chi}\right)$. Define the constant term of $E(g, \omega)$ by

$$
E^{B}(g, \omega)=\sum_{\chi} E^{B}\left(g, \Phi_{\chi}\right)
$$

where

$$
E^{B}\left(g, \Phi_{\chi}\right)=\int_{\underline{N}(\boldsymbol{A}) / \underline{N}(\boldsymbol{Q})} E\left(g n, \Phi_{\chi}\right) d n .
$$

Then Harder ([5]) has proved the following lemma.

LEMMA 6.2. If at $\lambda=\lambda_{0} E\left(g, \Phi_{\chi}\right)$ is holomorphic and $d E^{B}\left(g, \Phi_{\chi}\right)=0$, then the value of $E(g, \omega)$ at $\lambda=\lambda_{0}$ is a closed form. Moreover $E(g, \omega)$ and $E^{B}(g, \omega)$ represents the same cohomology class on the boundary $\partial(\bar{X} / \Gamma)$.

Next we calculate $E^{B}\left(g, \Phi_{\chi}\right)$.

\section{$\S 7$.}

For $\alpha \in \Psi$, let $\underline{G}_{\alpha}$ be the derived group of the centralizer in $\underline{G}$ of the connected component of the kernel of $\xi_{\alpha}$. Let $\underline{G}_{\alpha}(R)=K_{\alpha} A_{\alpha} N_{\alpha}$ be the Iwasawa decomposition (compatible with that of $\underline{G}(R)) ; N_{\alpha}=\underline{N}_{a}(R)$ where 
$\underline{N}_{\alpha}$ is a one parameter subgroup in $\underline{G}$. For $w \in W$, let

$$
\underline{N}^{w}=\prod_{\substack{\alpha \in W^{+} \\ w-1 \alpha \in \Psi_{+}^{+}}} \underline{N}_{\alpha} .
$$

Then we have the Bruhat decomposition

$$
\underline{G}(\boldsymbol{Q})=\bigcup_{w \in W} \underline{N}^{w}(\boldsymbol{Q}) r_{w} \underline{B}(\boldsymbol{Q})
$$

where $r_{w}$ belongs to the group of $\boldsymbol{Q}$-rational points of the normalizer of $\underline{T}$. Moreover $r_{w}$ can be chosen in $K$. We shall simply write $w$ for $r_{w}$. The following lemma is an easy consequence of the Bruhat decomposition (see for example [8] p. 85 and 277)

Lemma 7.1. For $g \in \underline{G}(A)$, let

$$
c(w, \lambda) \Phi(g)=\int_{\underline{N}^{w}(\boldsymbol{A})} \Phi(g n w) d n .
$$

Then

$$
E^{B}(g, \Phi)=\sum_{w \in W} c(w, \lambda) \Phi(g) .
$$

The integral in (7.1) actually gives the effect of the linear transformation $c(w, \lambda)$ on the function $\Phi$. For our purposes the exact space of functions (see [5] p. 149) on which $c(w, \lambda)$ acts is not important. However it is known that $c(w, \lambda)$ satisfies the following functional equation ([8] $\mathrm{p}$. 120)

$$
c\left(w_{1} w_{2}, \lambda\right)=c\left(w_{1}, w_{2} \lambda\right) c\left(w_{2}, \lambda\right)
$$

for $w_{1}, w_{2} \in W$. Since $W$ is generated by the reflections $w_{\alpha}$ for $\alpha \in \Delta$, the functional equation (7.3) allows us to restrict our attention to $c\left(w_{\alpha}, \lambda\right) \Phi(g)$ for $\alpha \in \Delta$. In fact it is sufficient to calculate $c\left(w_{\alpha}, \lambda\right) \Phi(t)$ for $t \in \underline{T}(\boldsymbol{A})$. In this case we have

$$
\begin{aligned}
c(w, \lambda) \Phi(t) & =\int_{\underline{\underline{N}} w(A)} \Phi\left(t n t^{-1} t w\right) d n \\
& =\xi_{\delta(w)}(t) \int_{\underline{N} w(A)} \Phi(n t w) d n
\end{aligned}
$$

where we have changed the variable once and used the fact that

$$
d\left(t n t^{-1}\right)=\xi_{\delta(w)}(t) d n
$$

with $\delta(w)=-\sum \alpha$ for $\alpha \in \Psi^{+}$and $w^{-1}(\alpha) \notin \Psi^{+}$. Put ${ }^{w} \chi(t)=\chi\left(w^{-1} t w\right)$. Using Lemma 5.1 (i), we get 


$$
\begin{aligned}
\Phi(n t w) & =\Phi\left(n w w^{-1} t w\right) \\
& =\Phi(n w)^{w} \chi(t) \xi_{-w \lambda-w \rho}(t)
\end{aligned}
$$

We also have

$$
\rho=w \rho-\delta(w) .
$$

Putting together these formulas, we get

$$
c(w, \lambda) \Phi(t)=\left(\int_{\underline{N}^{w}(\boldsymbol{A})} \Phi(n w) d n\right){ }^{w} \chi(t) \xi_{-\rho-w \lambda}(t) .
$$

Put $w_{\alpha j}=w_{j}$ and $\underline{N}_{j}=\underline{N}_{\alpha_{j}}$ for $\alpha_{j} \in \Delta$. Then $\underline{N}^{w} j=\underline{N}_{j}$. Choose a Haar measure $d x=\prod_{p} d x_{p}$ on $A$ such that $d x_{\infty}$ is the usual Euclidean measure on $\boldsymbol{R}$ and for all finite primes $q$, the volume of $Z_{q}$ with respect to $d x_{q}$ is 1. Identify the one parameter subgroup $\underline{N}_{j}(A)$ with $A$ and give it the Haar measure $d n$ induced from $d x$.

Let $\lambda_{i} \in \mathfrak{a}^{*}$ be defined by

$$
\frac{2\left(\lambda_{i}, \alpha_{j}\right)}{\left(\alpha_{j}, \alpha_{j}\right)}=\delta_{i j}
$$

where $\alpha_{1}, \cdots, \alpha_{\ell}$ are the simple roots (see $\S 1$ ) and $\delta_{i j}$ is the Kronecker delta. Then $\lambda_{1}, \cdots, \lambda_{l}$ are the fundamental dominant weights and every $\lambda \in \mathfrak{a}_{\boldsymbol{C}}^{*}$ can be written as $\sum s_{i} \lambda_{i}, s_{i} \in C$. For $t \in T(A)$, we have

$$
\xi_{-\lambda-\rho}(t)=\prod_{j=1}^{\ell}\left|\xi_{\lambda_{j}}(t)\right|_{A}^{-s_{j}-1}
$$

where $|\cdot|_{A}$ is the adelic norm. (Note that $\rho=\sum \lambda_{j}$ ).

Now we can return to the integral in (7.4). For $w=w_{j}$ we have

$$
\int_{\underline{N}_{j}(\boldsymbol{A})} \Phi\left(n w_{j}\right) d n=\operatorname{Lim}_{\mathscr{\varphi}} \int_{\underline{N}_{j}^{\varphi}} \Phi\left(n w_{j}\right) d n
$$

where $\mathscr{S}$ is a finite set of primes including infinity and the number of elements of $\mathscr{S}$ goes to infinity. $\underline{N}_{j}^{\varphi}$ is the subgroup of $\underline{N}_{j}(A)$ consisting of those $n=\left(n_{q}\right)$ in which $n_{q}=1$ for $q \notin \mathscr{S}$. This allows us to reduce the problem to the calculation of local factors. They are of three kinds. (In the following $\lambda=\sum s_{i} \lambda_{i}$ lies in the domain of convergence of the integral (7.7) and we apply Lemma 5.1)

The factor at infinity is

$$
\begin{aligned}
C_{\infty} & =\int_{\underline{N_{j}(\boldsymbol{R})}} \phi_{\lambda}\left(n_{\infty} w_{j}\right) d n_{\infty} \\
& =\int_{\underline{N_{i}(\boldsymbol{R})}} \Lambda^{m} \operatorname{Ad}^{*}\left(k\left(n_{\infty} w_{j}\right)\right) \phi(1) \prod_{i}\left|\xi_{\lambda_{i}}\left(a\left(n_{\infty} w_{j}\right)\right)\right|^{-s_{i}-1} d n_{\infty} .
\end{aligned}
$$


Here $n_{\infty} w_{j}=k\left(n_{\infty} w_{j}\right) a\left(n_{\infty} w_{j}\right) n\left(n_{\infty} w_{j}\right)$ (Iwasawa decomposition).

The factor at a finite prime $q \neq p$ is

$$
C_{q}=\int_{\underline{N_{j}\left(\boldsymbol{Q}_{q}\right)}} \chi\left(t\left(n_{q} w_{j}\right)\right) \prod_{i}\left|\xi_{\lambda_{i}}\left(t\left(n_{q} w_{j}\right)\right)\right|_{q}^{-s_{i}-1} d n_{q} .
$$

Here $n_{q} w_{j}=k\left(n_{q} w_{j}\right) t\left(n_{q} w_{j}\right) n\left(n_{q} w_{j}\right)$ (Iwasawa decomposition) and $|\cdot|_{q}$ is the valuation of $\boldsymbol{Q}_{q}$ such that $\left|\tilde{\omega}_{q}\right|_{q}=q^{-1}$ if $\tilde{\omega}_{q}$ is the uniformizing element of $\boldsymbol{Q}_{q}$. And finally the factor at $p$ is

$$
C_{p}=\int_{\underline{\underline{N}_{j}\left(\boldsymbol{Q}_{p}\right)}}\left(L\left(k\left(n_{p} w_{j}\right) f_{\chi}\right) \chi\left(t\left(n_{p} w_{j}\right)\right) \prod_{i}\left|\xi_{\lambda_{i}}\left(t\left(n_{p} w_{j}\right)\right)\right|_{p}^{-s_{i}-1} d n_{p}\right.
$$

where $n_{p} w_{j}=k\left(n_{p} w_{j}\right) t\left(n_{p} w_{j}\right) n\left(n_{p} w_{j}\right)$ is the Iwasawa decomposition of $n_{p} w_{j}$ in $\underline{G}\left(\boldsymbol{Q}_{p}\right)$.

$\S 8$.

To calculate the factor $C_{\infty}$ we need to know the explicit action of $\Lambda^{*} \mathrm{Ad}$.

We number the rows and columns of $2 \ell \times 2 \ell$ matrices by

$$
\{1,2, \cdots, \ell,-1,-2, \cdots,-\ell\} .
$$

Let $e_{i j}$ be the matrix which is 1 at $(i, j)^{\text {th }}$ entry and 0 elsewhere. For $\alpha_{j}$ $\in \Delta(1 \leq i \leq \ell-1)$ the Lie algebra $a_{j}$ of $A_{\alpha_{j}}$ is spanned by $e_{j}=e_{j j}-e_{j+1, j+1}$ $-e_{-j,-j}+e_{-j-1,-j-1}$. and the Lie algebra $a_{\ell}$ of $A_{\alpha_{\ell}}$ is spanned by $e_{\ell, \ell}-e_{-\ell, \ell}$. We write

$$
e_{\alpha}=\left\{\begin{array}{l}
e_{i j}-e_{-j,-i} \\
e_{j i}-e_{-i,-j} \\
e_{i,-j}+e_{j,-i} \\
e_{-i, j}+e_{-j, i} \\
e_{i,-i} \\
e_{-i, i}
\end{array} \quad \text { if } \alpha=\left\{\begin{array}{l}
\varepsilon_{i}-\varepsilon_{j} \\
\varepsilon_{j}-\varepsilon_{i} \\
\varepsilon_{i}+\varepsilon_{j} \\
-\varepsilon_{i}-\varepsilon_{j} \\
2 \varepsilon_{i} \\
-2 \varepsilon_{i}
\end{array} \quad(i<j)\right.\right.
$$

Then $e_{\alpha}$ is basis vector of $g_{\alpha}$. Let $\mathfrak{m}_{\alpha}$ be the space spanned by $e_{\alpha}+e_{-\alpha}$ and $\mathfrak{m}=\sum_{\alpha \in \Psi}+\mathfrak{m}_{\alpha}$. Then $\mathfrak{b}$ can be embedded in the space

$$
\mathfrak{p}=\sum_{\alpha_{j} \in \Delta} \mathfrak{a}_{j} \oplus \sum_{\alpha \in \Psi+} \mathfrak{m}_{\alpha} .
$$

If $1 \leq j \leq \ell-1$, we write 
$(8.2)$

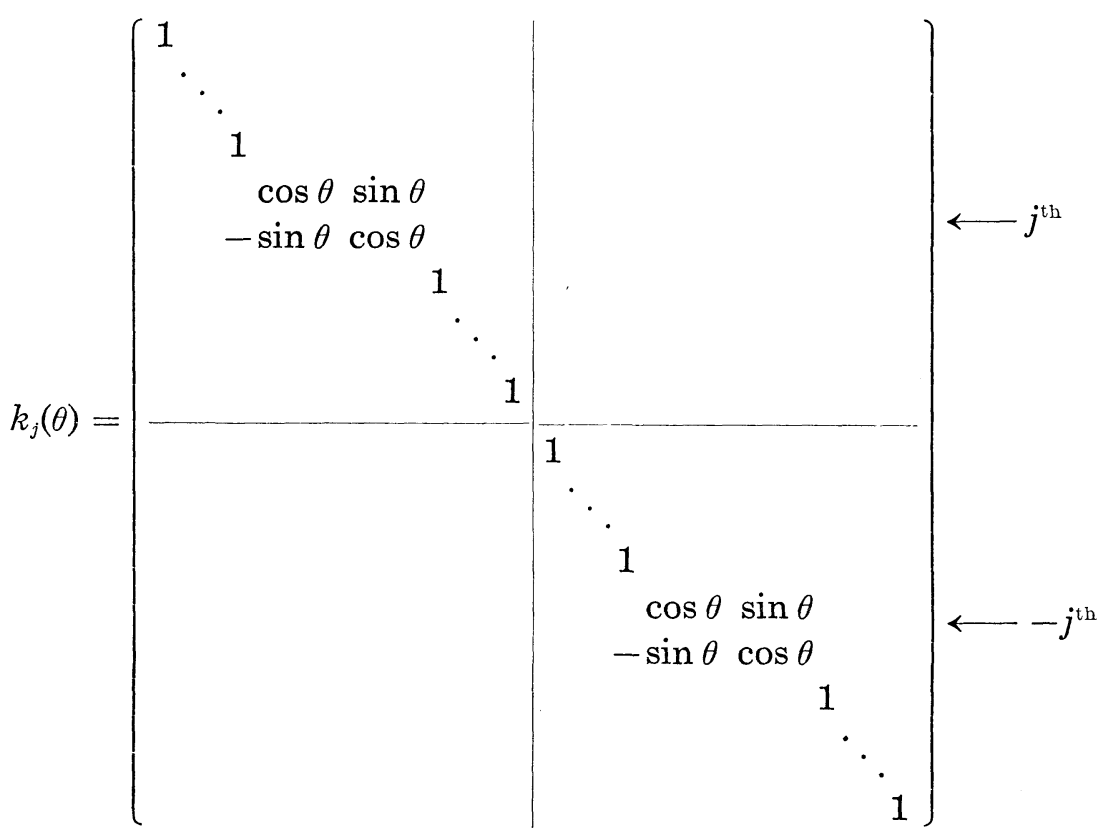

and

$$
k_{\ell}(\theta)=\left(\begin{array}{ll|ll}
I & & \\
& \cos \theta & \sin \theta \\
\hline-\sin \theta & \cos \theta
\end{array}\right),
$$

where $I$ is the $(\ell-1) \times(\ell-1)$ identity matrix.

Lemma 8.1. For $\alpha_{j} \in \Delta$, let $\mathrm{Ad}_{j}$ denotes the restriction to $K_{a_{j}}$ of the adjoin action of $K$ on $\mathfrak{p}$. Then we list below the $\mathrm{Ad}_{j}$ invariant subspaces together with the matrix of $\operatorname{Ad}_{j} k_{j}(\theta)$ with respect to the above basis.

(A) The case $\alpha_{j}=\varepsilon_{j}-\varepsilon_{j+1} \quad(1 \leq j \leq \ell)$

(1) $\mathfrak{a}_{j}+\mathfrak{m}_{\alpha_{j}}$; the matrix is

$$
\left(\begin{array}{rr}
\cos 2 \theta & \sin 2 \theta \\
-\sin 2 \theta & \cos 2 \theta
\end{array}\right)
$$

(2) $\mathfrak{m}_{2 \varepsilon_{j}}+\mathfrak{m}_{2 \varepsilon_{j+1}}+\mathfrak{m}_{\varepsilon_{j+\varepsilon_{j+1}}}$; the matrix is

$$
\left(\begin{array}{clr}
\cos ^{2} \theta & \sin ^{2} \theta & \sin 2 \theta \\
\sin ^{2} \theta & \cos ^{2} \theta & -\sin 2 \theta \\
-\sin \theta \cos \theta & \sin \theta \cos \theta & \cos 2 \theta
\end{array}\right)
$$

(3) $\mathfrak{m}_{\varepsilon_{j}-\varepsilon_{h}}+\mathfrak{m}_{\varepsilon_{j+1}-\varepsilon_{h}}$ for $j+2 \leq h \leq \ell$ 
(4) $\mathfrak{m}_{c_{j}+\varepsilon_{h}}+\mathfrak{m}_{c_{j+1}+\varepsilon_{h}} \quad$ for $1 \leq h \leq j-1$

(5) $\mathfrak{m}_{c_{j+\varepsilon_{h}}}+\mathfrak{m}_{c_{j+1}+\varepsilon_{h}} \quad$ for $j+2 \leq h \leq \ell$

(6) $\mathfrak{m}_{s_{h}-\varepsilon_{j}}+\mathfrak{m}_{s_{h-\varepsilon_{j}+1}} \quad$ for $1 \leq h \leq j-1$.

The matrix for the cases (3) to (6) is

$$
\left(\begin{array}{rr}
\cos \theta & \sin \theta \\
-\sin \theta & \cos \theta
\end{array}\right) \text {. }
$$

(7) $\mathfrak{m}_{\epsilon_{i \mp \epsilon_{h}}}$ for all pairs $(i, h)$ satisfying $i<h$ and either

$$
\begin{array}{lll} 
& 1 \leq i<j, & 2 \leq h<j ; \\
\text { or } & 1 \leq i<j, & j+1<h \leq \ell ; \\
\text { or } & j+1<i<\ell, & j+1<h \leq \ell .
\end{array}
$$

(8) $\mathfrak{m}_{2 \epsilon_{h}}$ for $1 \leq h \leq \ell$ and $h \neq j, \quad j+1$

$\operatorname{Ad}_{j} k_{j}(\theta)$ acts trivially on each of the spaces in (7) and (8).

(B) The case of $\alpha_{\ell}$

(1) $\mathfrak{a}_{\ell}+\mathfrak{m}_{\alpha_{\ell}}$; the matrix is

$$
\left(\begin{array}{rr}
\cos 2 \theta & \sin 2 \theta \\
-\sin 2 \theta & \cos 2 \theta
\end{array}\right)
$$

(2) $\mathfrak{m}_{e_{h-\varepsilon_{\ell}}}+\mathfrak{m}_{\varepsilon_{h}+\varepsilon_{\ell}}$ for $1 \leq h<\ell$; the matrix is

$$
\left(\begin{array}{rr}
\cos \theta & \sin \theta \\
-\sin \theta & \cos \theta
\end{array}\right)
$$

(3) $\mathfrak{m}_{c_{i \mp \iota_{h}}}$ for all pairs $(i, j)$ satisfying $i<h$ and $1 \leq i \leq \ell-2,2 \leq h$ $\leq \ell-1$

(4) $\mathfrak{m}_{2 \varepsilon_{h}}$ for $1 \leq h \leq \ell-1$

$\operatorname{Ad}_{j} k_{j}(\theta)$ acts trivially on each of the spaces in (3) and (4).

Corollary 8.2. Write $m=\operatorname{dim} \mathfrak{n}$ and $\mathfrak{e}=\Lambda_{\alpha \in \psi^{+}, \alpha \neq \alpha_{j}}\left(e_{\alpha}+e_{-\alpha}\right)$. Then $\Lambda^{m} \operatorname{Ad}_{j} k_{j}(\theta)$ acts on the 2 dimensional space

$$
\Lambda^{m}\left(\mathfrak{a}_{j} \oplus \sum_{\alpha \in \psi+, \alpha \neq \alpha_{j}} \mathfrak{m}_{\alpha}\right) \oplus \Lambda^{m} \mathfrak{m}
$$

by the matrix

$$
\left(\begin{array}{rr}
\cos 2 \theta & \sin 2 \theta \\
-\sin 2 \theta & \cos 2 \theta
\end{array}\right)
$$

with respect to the basis $\left\{e_{j} \wedge \mathfrak{e},\left(e_{\alpha_{j}}+e_{-\alpha_{j}}\right) \wedge \mathfrak{e}\right\}$.

The lemma is proved by means of a simple matrix calculation which will be omitted here. The corollary follows trivially from the lemma. 
If $\alpha_{j}=\varepsilon_{j}-\varepsilon_{j+1}, 1 \leq j \leq \ell-1$, then every element of $\underline{G}_{\alpha_{j}}(R)$ can be written as

$$
k_{j}(\theta) a_{t} n_{x}
$$

for some $\theta, x \in \boldsymbol{R}, t \in \boldsymbol{R}_{+}^{x}$; where $k_{j}(\theta)$ is given in $\S 8, n_{x}=I+x e_{a_{j}}$ and

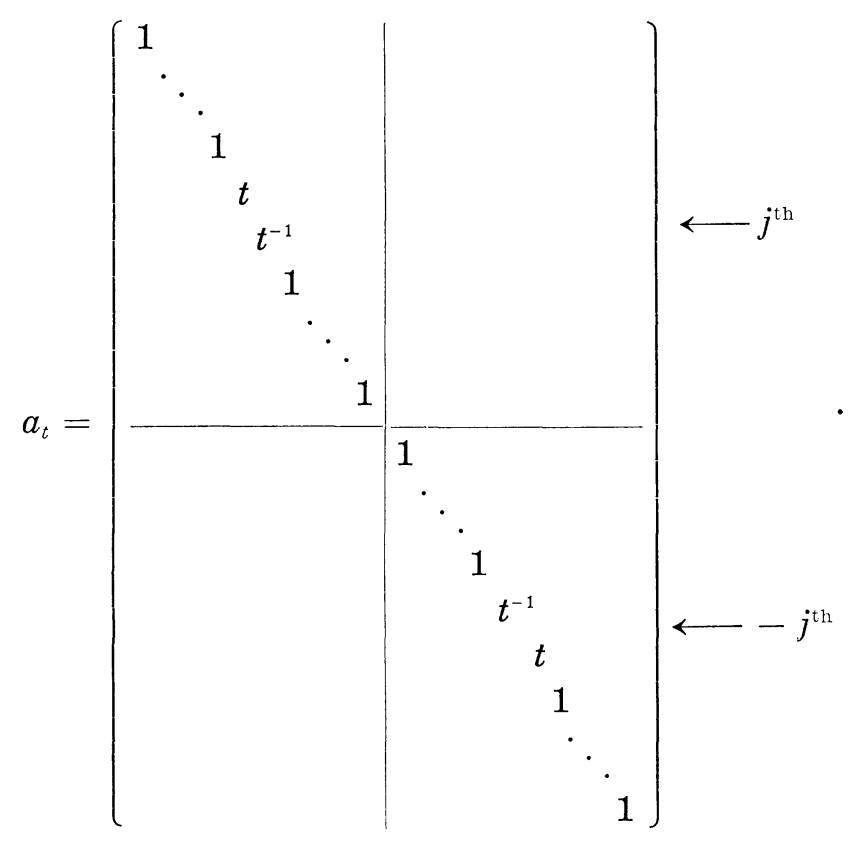

And in the case of $\alpha_{\ell}$, we have

$$
k_{\ell}(\theta) a_{t} n_{x} .
$$

Here $n_{x}=\left(I+x e_{\alpha_{\ell}}\right), k(\theta)$ is given in $\S 8$ and

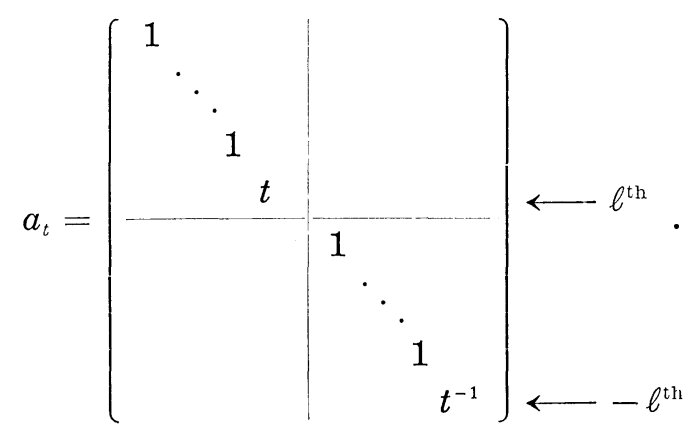

Moreover we put 


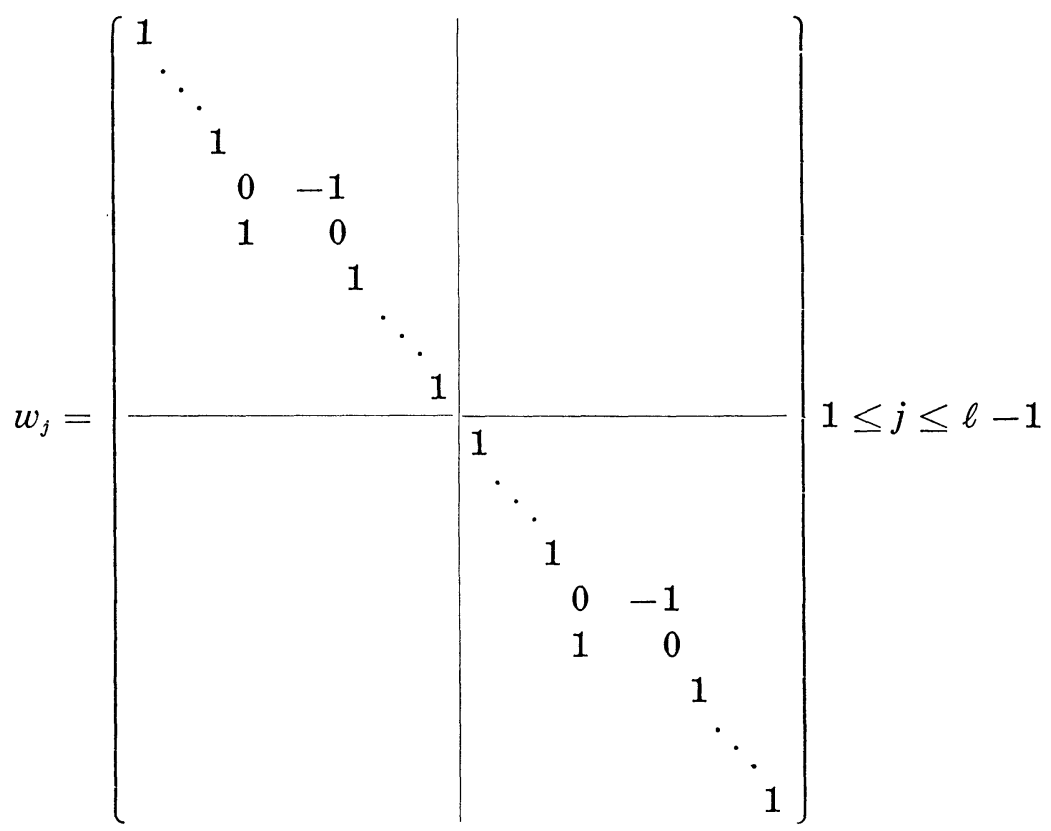

and

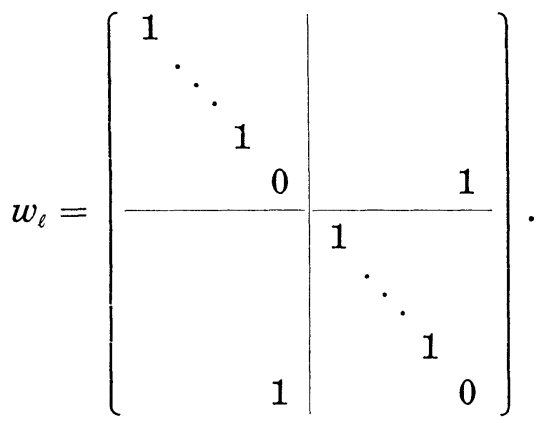

For $1 \leq j \leq \ell$, if the Iwasawa decomposition of $n_{x} w_{j}$ is $k_{j}(\theta) \cdot a_{t} \cdot n_{x^{\prime}}$ then

$$
x^{\prime}=-\frac{x}{1+x^{2}}, \quad t=\sqrt{1+x^{2}} \text { and } \sin \theta=\frac{-1}{\sqrt{1+x^{2}}}
$$

so that

$$
\prod_{i}\left|\xi_{\lambda_{i}}\left(a\left(n_{x} w_{j}\right)\right)\right|^{-s_{i}-1}=t^{-s_{j}-1} .
$$

By using Corollary 8.2, we see immediately from (7.8) that

$$
\begin{aligned}
C_{\infty} & =\left(\int_{R}\left(\begin{array}{rr}
\cos 2 \theta & \sin 2 \theta \\
-\sin 2 \theta & \cos 2 \theta
\end{array}\right) t^{-s_{j}-1} d x\right) \phi(1) \\
& =\left(\int_{R}\left(\begin{array}{ll}
x^{2}-1 & -2 x \\
2 x & x^{2}-1
\end{array}\right)\left(x^{2}+1\right)^{\left(-s_{j}-3\right) / 2} d x\right) \phi(1) .
\end{aligned}
$$


Evaluating the integrals in terms of $\Gamma$-functions we get

$$
C_{\infty}=\left(\frac{1-s_{j}}{1+s_{j}}\right) \frac{\Gamma(1 / 2) \Gamma\left(s_{j} / 2\right)}{\Gamma\left(\left(1+s_{j}\right) / 2\right)} \phi(1) .
$$

$\S 9$.

We compute in this section the factors at the finite primes. Put

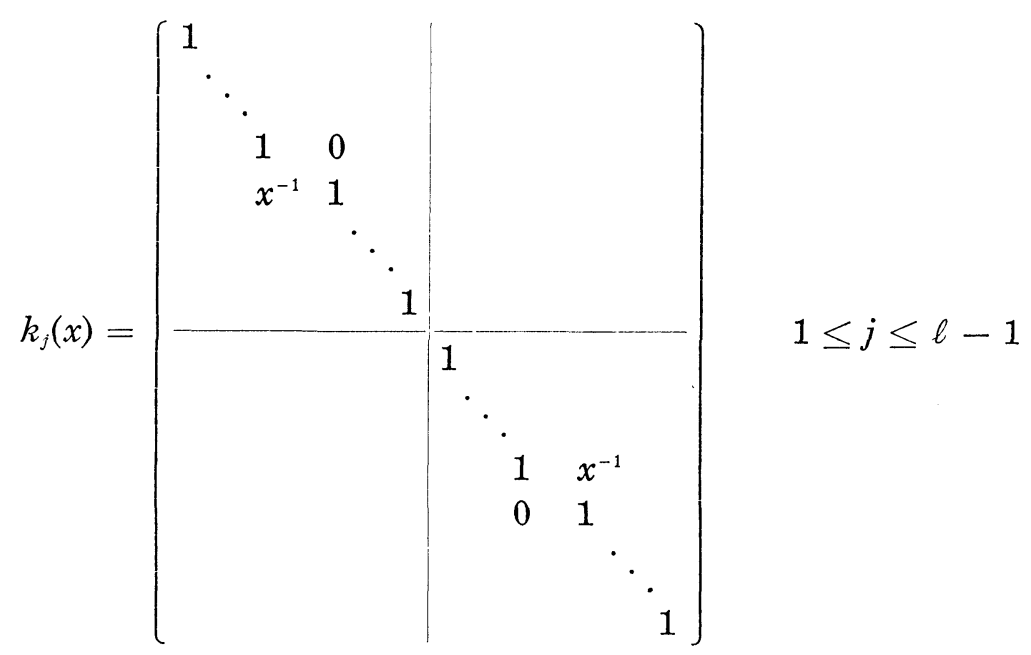

and

$$
k_{\ell}(x)=\left(\begin{array}{ll|ll}
I & & & \\
& 1 & & 0 \\
\hline & x^{-1} & & 1
\end{array}\right)
$$

where $I$ is the $(\ell-1) \times(\ell-1)$ identity matrix.

For a given $\alpha \in \Delta$, if $x \in \boldsymbol{Q}_{q}$ write $n_{x}=I+x e_{\alpha}$; if $t \in \boldsymbol{Q}_{q}^{\times}$, let $a_{t}$ be given by the matrix (8.4) (resp. (8.6)) in case $\alpha=\alpha_{j}, 1 \leq j \leq \ell-1$ (resp. $\alpha=\alpha_{\ell}$ ), then $T_{\alpha_{j}}\left(\boldsymbol{Q}_{q}\right)$ is just the set of matrices $a_{t}$. Let $\chi_{j}$ be the restriction of $\chi$ to $T_{\alpha_{j}}\left(\boldsymbol{Q}_{q}\right)$. Write $\chi_{j}\left(\tilde{\omega}_{q}\right)$ for $\chi_{j}\left(a_{\tilde{\omega}_{q}}\right)$. In this way we can regard $\chi_{j}$ as a character of $\boldsymbol{Q}_{q}$. We use the same $w_{j}$ as given in $\S 8$. Finally, if $x \in \boldsymbol{Q}_{q}^{\times}$, $\alpha=\alpha_{\jmath}$, then

$$
n_{x} w_{j}=k_{j}(x) a_{x} n_{-x-1} .
$$

First let us handle the case of $C_{q}, q \neq p$. Since $n_{x} w_{j} \in \underline{G}_{\alpha_{j}}\left(Z_{q}\right)$ if $x \in$ $\boldsymbol{Z}_{q}$, we have 


$$
\begin{aligned}
\prod_{i}\left|\xi_{i_{i}}\left(t\left(n_{x} w_{j}\right)\right)\right|_{q}^{-s_{i}-1} \\
\quad=\left\{\begin{array}{ll}
1 & \text { if } x \in Z_{q} \\
|x|_{q}^{-s_{j}-1} & \text { if } x \notin Z_{q}
\end{array} .\right.
\end{aligned}
$$

By the formula (7.9) $C_{q}$ equals to

$$
\begin{aligned}
& \int_{Z_{q}} d x+\sum_{m=1}^{\infty} \int_{\tilde{\omega}_{q}^{-m} Z_{q} \backslash \tilde{\omega}_{q}^{-(m-1)} Z_{q}} \chi_{j}\left(a_{x}\right)|x|_{q}^{-s_{j}-1} d x \\
& =\frac{1-\bar{\chi}_{j}\left(\tilde{\omega}_{q}\right)^{-\left(s_{j}+1\right)}}{1-\bar{\chi}_{j}\left(\tilde{\omega}_{q}\right) q^{-s_{j}}} .
\end{aligned}
$$

Let

$$
L(s, \chi)=\prod_{q}\left(1-\chi\left(\tilde{\omega}_{q}\right) q^{-s}\right)^{-1}
$$

be the Hecke $L$-function (the product is taken over all the finite primes $q$ where $\chi$ is unramified).

Let $\mathscr{S}_{j}$ be the set of finite primes at which $\chi_{j}$ is ramified. Let

$$
\kappa_{j}=\left\{\begin{array}{l}
\prod_{\left.q \in \mathscr{S}_{j} \backslash p\right\}} C_{q} \quad \text { if } p \in \mathscr{S}_{j} \\
\left(\prod_{q \in \mathscr{S}_{j}} C_{q}\right) \frac{1-\bar{\chi}_{j}\left(\tilde{\omega}_{p}\right) p^{-s_{j}}}{1-\bar{\chi}_{j}\left(\tilde{\omega}_{p}\right) p^{-\left(1+s_{j}\right)}} \quad \text { if } p \notin \mathscr{S}_{j} .
\end{array}\right.
$$

Then we get

$$
\prod_{q \neq p} C_{q}=\kappa_{j} \frac{L\left(s_{j}, \bar{\chi}_{j}\right)}{L\left(1+s_{j}, \bar{\chi}_{j}\right)} .
$$

(Note that the above formula was obtained under the assumption that $\lambda$ lies the domain of convergence of the integral (7.7). We can extend the formula to all $\lambda$ by the principle of analytic continuation.)

Next we consider the local factor $C_{p}$. According to Lemma 5.1, it is sufficient to study the integral

$$
\int_{\underline{N}_{j}\left(\boldsymbol{Q}_{p}\right)} \mid\left(\xi_{-\lambda-\rho}\left(t_{p}\left(n w_{j}\right)\right) \mid d n .\right.
$$

An easy calculation as above shows that (9.3) is equal to

$$
\frac{1-q^{-(\sigma+1)}}{1-q^{-\sigma}}, \quad \text { if } s_{j}=\sigma+\sqrt{-1} \tau \text {. }
$$

As a consequence we have the following

Lemma 9.1. $C_{p}$ is holomorphic in $\lambda=\sum s_{i} \lambda_{i}$ if $\operatorname{Re} s_{j}>0$. 
$\S 10$.

Suppose that under the map (3.3) the element $\omega$ of $\oplus_{P \in D} H^{m}\left(e^{\prime}(P), C\right)$ corresponds to $[\phi] \otimes \sum_{x} f_{\chi}$. If for each $\chi$ that appears the corresponding $\chi_{j} \neq 1$, then we say that $\omega$ is a regular class. Now we can state the theorem.

Theorem. If $\omega \in \oplus_{P \in D} H^{m}\left(e^{\prime}(P), C\right)$ is a regular class, then there exists $a \tilde{\omega} \in H^{*}(\bar{X} / \Gamma, C)$ such that $r(\tilde{\omega})=\omega$ ( $r$ is the restriction map (1.1)). Moreover $\tilde{\omega}$ can be represented by a harmonic form.

We first put together the results of the previous three sections on the constant term $E^{B}(g, \Phi)(6.9)$ of the Eisenstein series. We have

$$
E^{B}(g, \Phi)=\sum_{w \in W} c(w, \lambda) \Phi(g) .
$$

For $1 \leq j \leq \ell$ and $\lambda=\sum s_{i} \lambda_{i}, c\left(w_{j}, \lambda\right) \Phi$ is the product of

$$
\left(\phi(1) \otimes C_{P}\right) \chi^{w_{j}} \xi_{-\rho-w_{j} \lambda}
$$

and a constant

$$
\frac{1-s_{j}}{1+s_{j}} \frac{\Gamma(1 / 2) \Gamma\left(s_{j} / 2\right)}{\Gamma\left(\left(1+s_{j}\right) / 2\right)} \frac{L\left(s_{j}, \bar{\chi}_{j}\right)}{L\left(1+s_{\jmath}, \bar{\chi}_{\jmath}\right)} \kappa_{j}
$$

(cf: (7.4), (8.10) and (9.2)).

Now if $\chi$ is such that $\bar{\chi}_{j}$ is not the trivial character for all $j$, and $\lambda$ $=\sum \lambda_{i}=\rho$, then since $c\left(w_{j}, \lambda\right)$ is a linear transformation on function space (and is independent of $g$ ) it is clear from (10.2) that $c\left(w_{j}, \lambda\right) \phi$ is zero for all $j$. Moreover, as $c(w, \lambda) \Phi$ is holomorphic at $\rho$, the general properties of the transformations $c(w, \lambda)$ (cf: [8]) implies that the same is true for $c(w, \lambda) \Phi$. In fact the functional equation (7.3) implies that $c(w, \rho) \Phi$ is zero for all Weyl group elements $w$ which is not the identity. Thus at $\lambda=\rho$, we have

$$
E^{B}(g, \Phi)=c(\operatorname{Id}, \lambda) \Phi(g)=\Phi(g) .
$$

An easy calculation (using formula (2.3) of [5]) shows that

$$
d \Phi\left(H, X_{1}, \cdots, X_{m}\right)=(-\mu-\rho-\lambda) H \Phi\left(X_{1}, \cdots, X_{m}\right)
$$

for $H \in \mathfrak{a}, X_{1}, \cdots, X_{m} \in \mathfrak{n}$. According to (6.6), $\mu=-2 \rho$.

Hence at $\lambda=\rho$, we have

$$
d E^{B}(g, \Phi)=0
$$


and by (6.7),

$$
\Delta E(g, \phi)=0 \text {. }
$$

Suppose $[\phi] \otimes \sum_{x} f_{x}$ corresponds to the element $\omega$ in $\oplus_{P \in D} H^{*}\left(e^{\prime}(P), C\right)$. If $\omega$ is regular then $\chi_{j} \neq 1$ and by Lemma 4.1 of [8] $E\left(g, \Phi_{\chi}\right)$ is holomorphic at $\lambda=\rho$. Therefore by Lemma 6.2, (10.4), (10.5) the value of $E(g, \omega)$ at the special point $\lambda=\rho$ is an harmonic form representing a cohomology class $\tilde{\omega}$ and the restriction $r(\tilde{\omega})$ of $\tilde{\omega}$ to the boundary $\partial(\bar{X} / \Gamma)$ can be represented by $E^{B}(g, \omega)$. Hence by $(10.3), r(\tilde{\omega})=\omega$.

\section{REFERENCES}

[1] A. Borel, Some finiteness properties of adele groups over number fields, IHES Publ. Math., 16 (1963), 101-126.

[2] - - Cohomology of arithmetic groups, Proc. Int. Congress Math., Vancouver (1974) , 435-442.

[ 3 ] A. Borel and J. P. Serre, Corners and arithmetic groups, Comment. Math. Helvet., 48 (1973), 436-491.

[ 4 ] G. Harder, On the Cohomology of $S L_{2}(\theta)$, in Lie groups and their representations, Edited by I. M. Gelfand, London, (1975), 139-150.

[5] - On the Cohomology of discrete arithmetically defined groups, Proc. Int. Colloq. On Discrete Subgroups of Lie Groups and Applications to Moduli at Bombay (1973), Oxford University Press (1975), 129-160.

[6] Harish-Chandra, Automorphic forms on semi-simple Lie groups, Springer Lecture Notes in Math., 62 (1968).

[ 7 ] B. Kostant, Lie algebra cohomology and the generalized Borel-Weil theorem, Ann. of Math., 74 (1961), 329-387.

[8] R. P. Langlands, On the functional equations satisfied by Eisenstein series, Springer Lecture Notes in Math., 544 (1976).

[ 9 ] Y. Matsushima and S. Murakami, On vector bundle valued harmonic forms and automorphic forms on symmetric Riemannian manifolds, Ann. of Math., 78 (1963), 365-416.

[10] G. Schiffmann, Integrales d'entrelacement et fonctions de Whittaker, Bull. Soc. Math. France, 99 (1971), 3-72.

[11] J. Schwermer, Sur la cohomologie des sous-groupes de congruence de $S L_{3}(Z)$, C. R. Acad. Sc. Paris, 283 (1976), 817-820.

[12] J. Schwermer, Eisensteinreihen und die Kohomologie von Kongruenzuntergrupper von $S L_{n}(Z)$, Bonner Mathematische Schriften, 99 (1977).

[13] W. T. van Est, A generalization of the Cartan-Leray spectral sequence, Indag. Math., 20 (1958), 399-413.

[14] A. Well, Adeles and algebraic groupes, IAS Princeton, (1961).

Department of Mathematics

The Chinese University of Hong Kong

Hong Kong 